\title{
Design of Copolymeric Materials
}

\author{
Tanya Kurosky and J.M.Deutsch \\ University of California, Santa Cruz, U.S.A.
}

\begin{abstract}
We devise a method for designing materials that will have some desired structural characteristics. We apply it to multiblock copolymers that have two different types of monomers, A and B. We show how to determine what sequence of A's and B's should be synthesised in order to give a particular structure and morphology. Using this method in conjunction with the theory of microphase separation developed by Leibler, we show it is possible to efficiently search for a desired morphology. The method is quite general and can be extended to design isolated heteropolymers, such as proteins, with desired structural characteristics. We show that by making certain approximations to the exact algorithm, a method recently proposed by Shakhnovich and Gutin is obtained. The problems with this method are discussed and we propose an improved approximate algorithm that is computationally efficient.
\end{abstract}


The problem addressed in this letter is the following. Is there an efficient method for designing a material with a particular morphology or structure? We develop a systematic approach to this problem that we illustrate for the design of copolymeric materials.

Structures of single chains in solution have also been extensively studied, often in relation to the important biological question of how to determine the structure of a protein from its sequence [1, 2]. Work on the design of a chemical sequence which has a desired three dimensional structure has also been recently considered [3]. For the two dimensional model of Dill et al. 22 it has been possible to devise a set of rules that achieve a desired tertiary structure 酒. In three dimensions, much less is known. Ad hoc methods have been attempted [5, 6] but recent tests have shown that they are not entirely efficacious [7].

To illustrate our general method, we will apply it to design of block copolymers, which are polymers made out of more than one chemical species. We will consider copolymeric systems made up of two constituent types of monomers denoted A and B. The phase diagram of such materials has been studied as a function of the Flory interaction parameter $\chi$ and the lengths of the segments of A and B. Lamellar, hexagonally closed packed, body centred cubic [8], and gyroid [9] phases have been predicted. Experimentally copolymers have been found to exhibit a variety of different structures, sometimes referred to as microphases.

Suppose we would like to design a new phase that has a given symmetry. Until now, it was necessary to do an inefficient search through the phase diagram of the system in order to find the desired symmetry. We show below that we can find a function that when minimised can home in on the correct structure. We then implement this practically in the framework of the theory of copolymers developed by Leibler [8]. We then turn to the problem of protein design and discuss how the method we developed can be used in this context.

First we wish to determine the correct function to minimise in order to obtain the best sequence corresponding to the desired morphology. We begin with a formulation of the general problem that we wish to solve. Consider a system with coordinates denoted by $\Gamma$ and with a chemical sequence denoted by $S$. We can define a function which tells us whether each structure $\Gamma$ is in the desired set of structures. Call this $P_{\text {struct }}(\Gamma)$. It is a constant if $\Gamma$ belongs to the class of desired structures and 0 otherwise. In practice we will express $P_{\text {struct }}(\Gamma)$ in 
terms of the clamping potential defined below. Consider next the probability that a sequence $S$ gives a desired structure. Since this is a conditional probability we denote it by $P(S \mid$ struct $)$. We wish to find the maximum of $P(S \mid$ struct $)$ over all sequences $S$ keeping the structure fixed. To uniquely define $P(S \mid$ struct $)$, we have to choose the a priori probability of choosing an arbitrary sequence $S$. The simplest choice is that it is uniform, that is $P(S)$ is constant. We consider the system in equilibrium at a finite temperature so the probability that a sequence $S$ has structure $\Gamma$ is given by the Boltzmann factor $P(\Gamma \mid S)=\exp \left(-\left[H_{S}(\Gamma)-F_{o}(S)\right] / T\right)$, where $H_{S}(\Gamma)$ is the Hamiltonian of the system with a particular sequence $S$, and $F_{o}(S)$ is the corresponding free energy of the system with $S$ kept constant. Bayes' theorem states that the joint probability $P(\Gamma, S)=P(\Gamma \mid S) P(S)=P(S \mid \Gamma) P(\Gamma)$, hence after some algebra, we obtain

$$
P(S \mid \text { struct })=\sum_{\Gamma} P_{\text {struct }}(\Gamma) P(S \mid \Gamma)=\sum_{\Gamma} P_{\text {struct }}^{\prime}(\Gamma) \exp \left(-\left(H_{S}(\Gamma)-F_{o}(S)\right) / T\right)
$$

where

$$
P_{\text {struct }}^{\prime}(\Gamma)=\frac{P_{\text {struct }}(\Gamma)}{\sum_{S} \exp \left(-\left(H_{S}(\Gamma)-F_{o}(S)\right) / T\right)} .
$$

Because $P_{\text {struct }}^{\prime}$ does not depend on the sequence $S$, we can equally well regard it rather than $P_{\text {struct }}$ as given. It can be thought of as imposing an external clamping potential on the system, pushing it into the correct structure, $P_{\text {struct }}^{\prime}(\Gamma) \equiv \exp \left(-V_{\text {ext }}(\Gamma) / T\right)$. We will make an appropriate choice for the clamping potential, $V_{\text {ext }}(\Gamma)$ for each problem, based on physical reasoning rather than using (2) directly. Thus (11) can be further reduced to

$$
P(S \mid \text { struct }) \propto \exp \left(-\left(F_{\text {struct }}(S)-F_{o}(S)\right) / T\right) \equiv \exp (-\Delta F / T)
$$

where $F_{\text {struct }}(S)$ is the free energy pushed into a certain structure by the clamping potential

$$
\exp \left(-F_{\text {struct }}(S) / T\right) \equiv \sum_{\Gamma} \exp \left(\left(-H_{S}(\Gamma)+V_{\text {ext }}(\Gamma)\right) / T\right)
$$

The physical interpretation of this is clear. The optimum sequence is the one that minimises the difference $\Delta F$ between the unrestricted free energy and the free energy clamped in the desired structure. Intuitively this is reasonable because it picks out a sequence that naturally 
wants to spend a lot of time in this structure. This result is a generalisation of that used in determining couplings in Boltzmann machines [10].

As a first example, we apply the formalism above to design copolymeric systems with desired lattice structures. Leibler 8 has developed a theory of microphase separation for diblock copolymers. Here we have further generalised this to $m$ blocks each block being made up entirely of $\mathrm{A}$ monomers or of $\mathrm{B}$ monomers, the $i$ th having a length $l_{i}, i=1 \ldots m$. The fractional length of the ith block is $f_{i} \equiv l_{i} / L$ where $L$ is the total chain length. The A and B monomers have an incompatibility, or Flory, parameter $\chi$ giving the degree to which the $\mathrm{A}$ and $\mathrm{B}$ monomers wish to segregate. Leibler took as the order parameter $\Delta \rho$, the ensemble average of the difference between the density of $A$ 's and the average density of $A$ 's. He was able to construct an expansion of the free energy in terms of $\Delta \rho$ and calculated explicitly the expansion to fourth order in terms of the underlying chemical structure of the chains, that is, $l_{1}, l_{2}$, and $\chi$. This theory should work well near the spinodal point for this system, because $\Delta \rho$ is small there.

To determine the stability of density variations with different crystallographic symmetries, Leibler took $\Delta \rho$ to be a periodic function of position $\mathbf{r}$,

$$
\Delta \rho(\mathbf{r})=\sum_{j=1}^{n} \psi\left(\mathbf{q}_{\mathbf{j}}\right) \exp \left(i \mathbf{q}_{j} \cdot \mathbf{r}\right),
$$

choosing the $\mathbf{q}_{j}$ 's, $j=1, \ldots, n$ to be the smallest non-zero reciprocal lattice vectors of the lattice structure being considered. The magnitude, $q^{*}$, of the $\mathbf{q}_{j}$ 's is taken to be the wavevector at the spinodal point where divergent fluctuations first appear. He further took the magnitude, but not the phase, of all the $\psi\left(\mathbf{q}_{\mathbf{j}}\right)$ 's to be equal. By choosing different $\mathbf{q}_{j}$ 's and minimising the resultant free energies he computed which crystal structure had the lowest free energy. He was able to obtain a phase diagram for the system as a function of $f_{1}$ and $\chi N$. Besides the high temperature disordered phase he found that the body centred cubic, triangular (hex.), and lamellar (lam.) phases existed in different regions of the phase diagram. Further work 11, 12] extended this treatment to triblocks. 
It is convenient to take $V_{\text {ext }}(\Gamma)$ to be smoothly varying:

$$
V_{e x t}(\mathbf{r})=-v \sum_{j=1}^{n} \exp \left(i \mathbf{q}_{j} \cdot \mathbf{r}\right) .
$$

The magnitude of $v$ adjusts the degree to which $\Delta \rho$ fluctuates. A clamping potential with, for example, hexagonal symmetry is the sum of three plane waves. Applying such a potential to the unclamped free energy $F_{0}$, we obtain a clamped free energy

$$
F\left(V_{e x t}\right)=F_{0}+\int \rho(\mathbf{r}) V_{e x t}(\mathbf{r}) d^{3} r
$$

which will tend to push the system into a phase with the symmetry of the external potential.

Unfortunately in the general formalism developed above it is necessary that the clamping potential $V_{\text {ext }}$ is very large when monomers stray from the desired structure. This requirement leads to a large $\Delta \rho$ and hence is incompatible with the limits of validity of the free energy expansion of Leibler which is only valid in the limit of weak segregation. Therefore we need to consider complications that arise when only a weak clamping potential is applied.

For small $V_{\text {ext }}$, the system will not always be pushed into the symmetry of the external potential. In fact, for the values of $v$ that we use, the effect of adding $V_{\text {ext }}$ is only to slightly enlarge the region of the phase diagram that has the symmetry of $V_{\text {ext }}$. Our algorithm determines which phase the system is in by allowing the magnitudes of the $\psi\left(\mathbf{q}_{\mathbf{j}}\right)$ 's to be unequal and then minimising with respect to them. This allows the possibility of mixed phases with more than one type of symmetry. For example if we consider the hexagonal phase and write (5) in terms of its wave vectors $\mathbf{q}_{1}, \mathbf{q}_{2}$, and $\mathbf{q}_{3}$,

$$
\Delta \rho(\mathbf{r})=\psi_{\text {lam }} \exp \left(i \mathbf{q}_{1} \cdot \mathbf{r}\right)+\psi_{\text {hex }}\left(\exp \left(i \mathbf{q}_{2} \cdot \mathbf{r}\right)+\exp \left(i \mathbf{q}_{3} \cdot \mathbf{r}\right)\right)
$$

then for $\psi_{\text {lam }}=\psi_{\text {hex }}$ we have a hexagonal structure $(n=3$ for the hex. phase), and for $\psi_{\text {hex }}=0$ we have a lamellar structure ( $n=1$ for the lam. phase).

For small $V_{\text {ext }}$,

$$
\Delta F \equiv F\left(V_{e x t}\right)-F_{0}=v(\partial \Delta F / \partial v)=-v \sum_{j=1}^{n_{c}} \psi\left(\mathbf{q}_{\mathbf{j}}\right)
$$


where $n_{c}$ is the number of $\mathbf{q}_{\mathbf{j}}$ ') s that $V_{\text {ext }}$ has in common with $\Delta \rho(\mathbf{r})$. From this it can be seen that $\Delta F$ does not do quite what one would like. In general a clamping potential will have reciprocal lattice vectors in common with more than one type of symmetry in $\Delta \rho(\mathbf{r})$. This means that $\Delta F$ will be lowered for other phases in addition to the one with the symmetry of $V_{\text {ext }}$. For example, for a clamping potential with hexagonal symmetry $V_{\text {hex }}, \Delta F\left(V_{\text {hex }}\right)=$ $F\left(V_{h e x}\right)-F_{0}$ will be lowered for the lamellar phase as well as the hexagonal. We would like a functional which is more selective in order to design a hexagonal material. The solution in this case is to subtract the lamellar component as follows. Looking at (8), we see that the appropriate functional to maximise is $\psi_{h e x}$. We would like to express $\psi_{h e x}$ in terms of free energy differences. From (9) $\left.\psi_{\text {hex }}=-\left(\partial \Delta F\left(V_{\text {hex }}\right) / \partial v\right)-\partial \Delta F\left(V_{l a m}\right) / \partial v\right) / 2$. For small $v$ this is proportional to $F\left(V_{h e x}\right)-F\left(V_{l a m}\right)$. Therefore minimising the difference in free energy between hexagonal and lamellar clamping will bring the system into the hexagonal phase. Fig. 11(a) shows $\left(F\left(V_{\text {hex }}\right)-F\left(V_{\text {lam }}\right)\right)$ as a function of the fraction $f$ for diblock copolymers at $\chi=20$. The minimum $f=0.32$ occurs in the correct position indicated by Leibler's theory.

We also tested out this method for designing diblock bcc structures. Fig. 1 (b) shows $F\left(V_{b c c}\right)-F\left(V_{h e x}\right)$ as a function of $f$ at $\chi=20$. The minimum $f=.23$ also occurs in the correct position but there is also a secondary small minimum at $f=.32$. This shows that there is no guarantee that there will only be one minimum for this minimisation function.

Now we turn to the problem of protein design. An interesting approach has recently been proposed by Shakhnovich and Gutin [5, [6] (SG), who proposed a method for solving this problem for a simplified lattice model using a self avoiding chain. The model they employed has sequences $\left\{\sigma_{i}\right\}$ of two possible monomer types that are given values \pm 1 , for chains of length $N$. This, plus the positions of all the monomers $\left\{r_{i}\right\}$, completely describe the state of the chain. We wish to find a sequence that causes the chain to fold up into a desired structure, but we do not care about what the monomer types end up being. The energy is

$$
E\left(\left\{\sigma_{i}\right\},\left\{r_{i}\right\}\right)=\frac{1}{2} \sum_{i, j}^{N}\left(B_{0}+B \sigma_{i} \sigma_{j}\right) \Delta\left(\mathbf{r}_{i}-\mathbf{r}_{j}\right)
$$

Here we will consider the case where $\Delta\left(\mathbf{r}_{i}-\mathbf{r}_{j}\right)=1$ if $\mathbf{r}_{i}$ and $\mathbf{r}_{j}$ are nearest neighbours, and is zero otherwise. One sets $B<0$, since this favours ferromagnetic ordering of the $\sigma$ 's, which 
means that the monomers will want to segregate, and $B_{0}<0$ since this provides an attractive interaction between monomers causing the protein to collapse.

Their method of sequence determination was to do a constrained minimisation of the energy of the chain in sequence space. The constraint was that the total magnetisation was held constant, in practice, close to zero. Note that without this constraint, all the $\sigma$ 's would become equal, and one would have a homopolymer which does not have a well defined structure. Unfortunately it appears that even with constrained minimisation, sequences found do not necessarily have to have the desired structures as the lowest energy states[0]. Even if the desired structure is a ground state, there may be a large ground state degeneracy in which case the structure is ill defined, as in the case of the unconstrained minimisation just mentioned.

The correct functional to minimise is $\Delta F$ defined in (3). If we specialise to the problem considered here, the constraining potential is a delta function since our structure is to be precisely determined. Calling the coordinates of the desired structure $\left\{r_{i}^{0}\right\}$, the correct functional to minimise is, according to (3),

$$
\Delta F=E\left(\left\{\sigma_{i}\right\},\left\{r_{i}^{0}\right\}\right)-F_{o}\left(\left\{\sigma_{i}\right\}\right) .
$$

SG's method is a minimisation of only the first term, with a constraint of constant total magnetisation. We will now argue that second term is not negligible and cannot be omitted. In fact, we will see that a crude approximation gives an answer similar to the constraint of constant magnetisation. But this approximation is of dubious validity which is why it failed.

Take $B_{0}$ to be very large and negative so that all stable structures must be globular with minimal surface area. The coordinates of our structure $\left\{r_{i}^{0}\right\}$ are therefore constrained to be of this compact type and the term in the energy involving $B_{0}$ will not vary and can now be ignored in the minimisation. The space of all conformations $\left\{r_{i}\right\}$ we need to consider are also compact conformations of the same overall shape, but different internal arrangements. We now expand out $F\left(\left\{\sigma_{i}\right\}\right)$ keeping only the lowest order cumulant. $F\left(\left\{\sigma_{i}\right\}\right) \approx\langle E\rangle+$ constant. The angled brackets denote an average that is equally weighted over all compact conformations with minimal surface area. Ignoring constant terms, this gives $F_{o}\left(\left\{\sigma_{i}\right\}\right) \approx \frac{B}{2} \sum_{i, j}^{N} \sigma_{i} \sigma_{j}\left\langle\Delta\left(\mathbf{r}_{i}-\mathbf{r}_{j}\right)\right\rangle$ 
SO

$$
\Delta F \approx \frac{B}{2} \sum_{i, j}^{N}\left[\Delta\left(\mathbf{r}_{i}-\mathbf{r}_{j}\right)-\left\langle\Delta\left(\mathbf{r}_{i}-\mathbf{r}_{j}\right)\right\rangle\right] \sigma_{i} \sigma_{j}
$$

One can easily show that the nearest neighbour interactions along the backbone of the chain cancel, because the probability that monomers $i$ and $i+1$ are next to each other is unity. This must happen because this kind of interaction is the same for all configurations and consequently cannot play a role in choosing the optimum $\sigma$ 's. We now find an approximate functional form for $\left\langle\Delta\left(\mathbf{r}_{i}-\mathbf{r}_{j}\right)\right\rangle$. Since the chain is compact there is a short screening length. We therefore expect random walk correlations when $|i-j|$ is greater than a few lattice spacings. However when $|i-j|^{1 / 2}$ becomes of order the diameter of the protein, the conformations cease to look like random walks as the protein is compact. This crossover corresponds to $|i-j| \sim N^{2 / 3}$. For scales larger than this the correlation function should be almost constant. Therefore

$$
\left\langle\Delta\left(\mathbf{r}_{i}-\mathbf{r}_{j}\right)\right\rangle \sim\left\{\begin{array}{l}
|i-j|^{-3 / 2} \text { for } 1<<|i-j|<<N^{2 / 3} \\
1 / N \text { for }|i-j|>>N^{2 / 3} .
\end{array}\right.
$$

Therefore in this approximation, $F\left(\left\{\sigma_{i}\right\}\right)$ looks like a one dimensional Ising model with the above long range interaction.

If we ignore the variation of $\left\langle\Delta\left(\mathbf{r}_{i}-\mathbf{r}_{j}\right)\right\rangle$ with $|i-j|$, i.e. $\left\langle\Delta\left(\mathbf{r}_{i}-\mathbf{r}_{j}\right)\right\rangle=1 / N$ so $\Delta F_{M F}=$ $E\left(\left\{\sigma_{i}\right\},\left\{r_{i}^{0}\right\}\right)-B\left(\sum_{i}^{N} \sigma_{i}\right)^{2} /(2 N)$, then $F_{o}$ gives an infinite range mean field contribution that is antiferromagnetic, and hence acts as a "soft" constraint favouring a total magnetisation of zero. To have a total magnetisation of zero, one must introduce a domain wall, which increases $E\left(\left\{\sigma_{i}\right\},\left\{r_{i}^{0}\right\}\right)$ by of order $N^{2 / 3}$, but this is more than compensated by the gain in free energy of the second term which is of order $N$. Hence in this limit we recover the approximation of SG. However this is a rather drastic approximation. Even within the first order expansion derived above, one is not justified in neglecting the shorter distance variation of $\left\langle\Delta\left(\mathbf{r}_{i}-\mathbf{r}_{j}\right)\right\rangle$ because, within this mean field approximation, there is a spurious degeneracy in the $\sigma$ 's that minimise $\Delta F_{M F}$. This is because the three dimensional arrangement of the $\sigma_{i}$ 's namely $\sigma(\mathbf{r})$ is independent of the desired conformation $\left\{r_{i}^{0}\right\}$. That is, to find the correct sequence, it is not necessary to consider the different internal arrangements of the chain inside the compact cluster, as they all give an identical $\Delta F_{M F}$. After the minimisation of $\Delta F_{M F}$ has 
been performed once, the three dimensional arrangement of the $\sigma$ 's do not change when the desired conformation is changed.

However a non-constant $\left\langle\Delta\left(\mathbf{r}_{i}-\mathbf{r}_{j}\right)\right\rangle$ breaks this degeneracy. It adds antiferromagnetic couplings along the backbone of the chain. This means that the domain wall should tend to orient itself roughly perpendicular to the direction of the backbone of the chain to satisfy the antiferromagnetic couplings. The contribution to $\Delta F$ due to the $|i-j|^{-3 / 2}$ decay of $\left\langle\Delta\left(\mathbf{r}_{i}-\mathbf{r}_{j}\right)\right\rangle$ is substantial and cannot be neglected. We can easily estimate it for the case of a desired structure $\left\{r_{i}^{0}\right\}$ that is in a typical random configuration. For a piece of arclength $s \equiv|i-j|=N^{2 / 3}$ the contribution to $\Delta F$ is of order $B s^{2} / s^{3 / 2}=B L^{1 / 3}$. But there are $L / L^{2 / 3}$ such pieces, giving a total contribution of order $B L^{2 / 3}$. This precisely the same order as the energy of the domain wall and therefore must be considered in doing protein design.

In conclusion, we have developed a method to design molecules that will self assemble into a desired structure. We used this to design block copolymers with desired structural characteristics, within the framework of Leibler's mean field theory.

We also note that the method for design described above should also work for the problem of protein design, for which no trustworthy methods have been devised so far. It would be interesting to see how well the approximate minimisation function, given by (12), designs stable proteins.

One of us (T.K.) wishes to thank Mark Dinan, Hemant Bokil and Doug Williams for useful discussions. We would also like to thank A.P. Young for useful comments and a critical reading of the manuscript. This work is supported by NSF grant number DMR-9419362 and acknowledgement is made to the Donors of the Petroleum Research Fund, administered by the American Chemical Society for partial support of this research. 


\section{References}

[1] Creighton T E 1993 Proteins : structures and molecular properties 2nd edn (New York, W.H. Freeman)

[2] Dill K A 1990 Biochemistry 297133

[3] Chan H S and Dill K A 1991 J. Chem. Phys. 953775

[4] Yue K and Dill K A 1992 Proc. Nat. Acad. Sci. (USA) 894163

[5] Shakhnovich E I, Gutin A M 1993 Protein Eng. 6793

[6] Shakhnovich E I, Gutin A M 1993 Proc. Nat. Acad. Sci. (USA) 9071956793

[7] Yue K, Fiebig K M, Thomas P D, Chan H S, E.I. Shakhnovich and Dill K A 1995 Proc. Nat. Acad. Sci. (USA) 92325

[8] Leibler L 1980 Macromol 131602

[9] Matsen M W and Schick M 1994 Phys. Rev. Lett. 722660

[10] Hertz J, Krogh A, and Palmer R G, 1991 Introduction to the theory of neural computation (Addison-Wesley Pub Co)

[11] Mayes A M and Olvera de la Cruz M. 1989 J. Chem. Phys. 917228

[12] Olvera de la Cruz M. 1989 J. Chem. Phys. 901995 


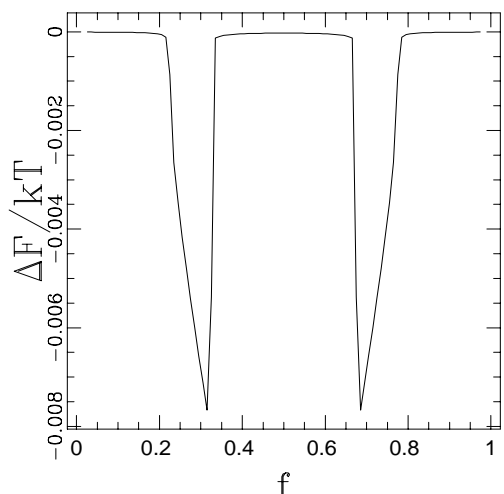

(a)

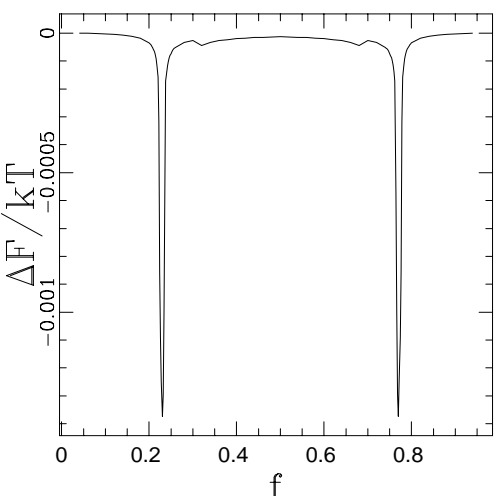

(b)

Figure 1: (a) $F\left(V_{h e x}\right)-F\left(V_{l a m}\right)$ and $(\mathrm{b}) F\left(V_{b c c}\right)-F\left(V_{h e x}\right)$, as a function of the fraction $f$ for diblock copolymer design. The minima give the best choice for the design of hexagonal material and bcc material, respectively. 